\title{
Effects of Antipsychotic Drugs on Neuroactive Steroids Brain and Plasma Levels in Humans and Animals: A Systematic Review of the Literature
}

\author{
Emerson A Nunes ${ }^{1 *}$, Joao Paulo Maia De Oliveira ${ }^{2}$, Glen B Baker ${ }^{3}$ and Jaime EC Hallak ${ }^{4}$ \\ ${ }^{1}$ Onofre Lopes University Hospital, UFRN, Brazil \\ ${ }^{2}$ Department of Clinical Medicine, UFRN, Brazil \\ ${ }^{3}$ Department of Psychiatry University of Alberta, Neurochemical Research Unit, Edmonton, Canada \\ ${ }^{4}$ Department of Neuroscience and Behavior, University of São Paulo,Brazil
}

*Corresponding author: Emerson A Nunes, Department of Neuroscience and

Behavior, University of São Paulo, Brazil.

Received Date: September 17, 2019

Published Date: September 26, 2019

\begin{abstract}
Objectives: The present study aims to review systematically the studies that investigated the effects of antipsychotic drugs on neuroactive steroids brain and plasma levels in animal and human studies

Methods: We conducted a review of the Medline databases, including articles published in English, Spanish and French, describing the results of controlled original animal and human studies that evaluated the effects of antipsychotics on brain and plasma neuroactive steroids levels.

Results: It was identified 291 studies through our search strategy. Of these studies, we selected 20, with one more study included after further searching throughout the reference list. Of these 21 studies, eight studies evaluated neuroactive steroids levels in humans, and 13 were animal studies. The antipsychotic used in the studies were: haloperidol, sulpiride, clozapine, olanzapine, risperidone, quetiapine and aripiprazol. Among the neuroactive steroids evaluated, we found studies evaluating levels of progesterone, allopregnanolone, DHEA, DHEA-S, TDHOC, pregnenolone and 3a,5a-THP. The human studies demonstrated diverse and non-conclusive alterations on the plasma levels of progesterone, no changes on allopregnanolone and TDHOC levels after clozapine use. Among the animal studies, nine of these evaluated progesterone levels, with no changes in three studies, and increased plasma levels in six studies. The same researchers in two studies investigated pregnenolone brain levels in rats, showing that clozapine and olanzapine acutely increased the levels of pregnenolone in rats. Four studies evaluating allopregnanolone showed increased brain levels after the use of olanzapine and clozapine. No changes with haloperidol or risperidone. The only animal study that investigated DHEA/DHEA-S levels, showed that clozapine decreased brain levels of DHEA-S, unlike haloperidol. Increased TDHOC levels after use of clozapine were observed in the only study that measured this neuroactive steroid. Two studies from the same group showed increased brain levels of 3a,5a-THP after olanzapine use.

Conclusion: The antipsychotic effects observed on neuroactive steroids levels reinforce their potential role in the pathophysiology of mental disorders, particularly schizophrenia. In the case of progesterone, the studies are in sufficient number to demonstrate the effect of antipsychotic on changes in their levels in animal but human studies. For the other neuroactive steroids, the inconclusive data points to the need for more well designed studies.
\end{abstract}

\section{Introduction}

Schizophrenia follows as one of the most challenge mental disorders, despite the highly available pharmacological treatment options, currently represented mainly by antipsychotic medications [1,2]. Most of these antipsychotic medications have in common some level of antagonism of dopamine type 2 receptors (D2) and also blockade of 5HT2A serotonin receptors is present in some of these compounds [3,4]. Even with the different types of currently available antipsychotics, all of them as stated earlier, have in common some blockade of D2 receptors. This pharmacodynamics effect is in accordance to the main theory that tries to explain the physiopathology of schizophrenia, the dopaminergic theory (CARLSSON AND LINDQVIST, 1963). Albeit reasonable improvement can be achieved with the use of antipsychotic to treat schizophrenia symptoms, especially when we consider the positive symptoms (hallucinations and delusions), almost one third of all patients is completely refractory to pharmacological approaches $[1,5]$. Therefore, the need for new drugs that target other neurotransmission systems other than the dopamine transmission is urgent [6] and the neuroactive steroids, like pregnenolone glutamate has attracted great attention 
in the last decade (MARX et al., 2011). Neuroactive steroids are endogenous molecules derived from cholesterol metabolism, which exert actions in the central nervous system. They may also suffer biotransformation in the central nervous system, only then reaching biological action, when they are called neurosteroids (NOORBAKHSH et al., 2011). Neuroactive steroids, including testosterone, dehydroepiandrosterone (DHEA) and its sulfate (DHEA-S) might play an important role in the pathophysiology of schizophrenia [7]. Many of the neuroactive steroids demonstrate important pleiotropic actions that significantly change brain function in animal models, these include modulation of GABAergic inhibitory and excitatory glutamatergic systems (Wu et al., 1991; Paul and Purdy, 1992; Belelli AND Lambert, 2005; Belelli et al., 2006), increased neurogenesis (MAYO et al., 2005), dendritic growth (FONTAINE-LENOIR et al., 2006), and myelination (KOENIG et al, 1995; . Azcoitia et al. 2003; GHOUMARI et al. 2003. LIAO et al, 2009), among other functions (MARX et al, 2011) [8]. Given the pleiotropic range of actions performed by different neuroactive steroids, demonstrated in animal models, it is assumed that probably deregulation of such substances should be relevant to the pathophysiology and treatment of various mental disorders, and that neuroactive steroids can be seen as potential new therapeutic agents.

Data from different studies in animal models have demonstrated the potential of such steroids as important factors in the pathophysiology of different mental disorders. We can mention the effects of allopregnanolone, a neuroactive GABAergic steroid, which has shown positive results in animal models of schizophrenia (reversing cognitive effects of MK-801, an animal model of schizophrenia) (ROMEO et al., 1994; Cheney et al. 1995), as well as in studies with animal models of other mental disorders such as post-traumatic stress disorder, depressive disorder and pain models (Pibiri et al, 2008; PINNA, 2010; MATSUMOTO et al, 2005; AGÍS- BALBOA et al, 2007; Meyer et al, 2011). Pregnenolone, a neuroactive steroid from the cholesterol metabolism, a progesterone precursor, have demonstrated positive properties on memory and learning in animal models in mice (Flood et al, 1992). Alopregnenolona, another neuroactive steroid from the same metabolic chain, has shown potentiating action on the action of GABA A receptors, exhibiting neuroprotective properties in animal models [9]. Thus, it is suggested that the pathways for biosynthesis involving the formation of pregnenolone, alopregnanolone and sulfate alopregnanolone, should be relevant to the pathophysiology and treatment of schizophrenia, worth note that these substances are interconverted, becoming complex understanding what to expect when trying to manipulate the concentrations of these molecules when administered exogenously, or while trying to accurately measure their serum or brain levels (these in animal models) (Marx et al., 2011). Therefore, antipsychotic drugs already used to treat schizophrenia may possibly have mechanism of action not only influencing directly the dopamine and serotonin receptors, as was recognized earlier, but also influencing GABAergic action, interfering with brain and serum levels of neuroactive steroids. Changes in the levels of neuroactive steroids (progesterone and alopregnenolona) have been observed after the use of olanzapine and clozapine in rats [9], reinforcing the theory of an influence of neuroactive steroids in the mechanism of action of drugs that target the symptoms present in schizophrenia. Thus, the present study aims to systematically review the studies that investigated the effects of antipsychotic drugs on neuroactive steroids serum and brain levels in animal and human studies.

\section{Methods}

A systematic review of the Medline database was conducted using a search strategy that combined the following keywords: (antipsychotic* or neuroleptic* or ziprasidone or haloperidol or thioridazine or clozapine or olanzapine or quetiapine or sulpiride or amisulpride or risperidone or fluphenazine or chlorpromazine) and (neurosteroid* or neuroactive steroid* or pregnenolone or "pregnenolone sulfate" or pregnanolone or dehidroepiandrostenedione or "dehidroepiandrostenedione sulfate" or progesterone or allopregnanolone or "allopregnanolone sulfate") and ("brain levels" or levels or "plasma level*" or "tissue levels" or "serum levels" or "cerebrospinal fluid level*" or "CSF levels"). It was included articles published in English, Spanish and French, describing the results of original human and animal studies that evaluated the effects of antipsychotics on serum and brain levels of neuroactive steroids. Also it was required as an inclusion criteria the presence of a control group. The publication period included articles published in the last 20 years. Review articles, letters to the editor, case reports and other publications that present no direct empirical results were all excluded.

\section{Results}

Table 1:

\begin{tabular}{|c|c|c|c|c|c|c|c|c|c|c|c|c|c|}
\hline Study & $\begin{array}{l}\text { Cou- } \\
\text { ntry }\end{array}$ & $\begin{array}{l}\text { Con- } \\
\text { trol }\end{array}$ & $\begin{array}{l}\text { Study } \\
\text { design }\end{array}$ & Subjects & $\mathbf{N}$ & Drug & $\begin{array}{l}\text { Ad- } \\
\text { mini- } \\
\text { stra- } \\
\text { tion }\end{array}$ & $\begin{array}{c}\text { Dura- } \\
\text { tion }\end{array}$ & $\begin{array}{l}\text { Neuro- } \\
\text { active } \\
\text { steroid }\end{array}$ & $\begin{array}{l}\text { Tis- } \\
\text { sue }\end{array}$ & $\begin{array}{l}\text { Re- } \\
\text { gion }\end{array}$ & Essay & Results \\
\hline $\begin{array}{l}\text { Mosta- } \\
\text { fapour } \\
\text { et al. } \\
2014\end{array}$ & Iran & Yes & $\begin{array}{l}\text { Longitu- } \\
\text { dinal } \\
\text { Animal, } \\
\text { con- } \\
\text { trolled }\end{array}$ & Wistar rats & 40 & $\begin{array}{l}\text { Sulpiride } \\
20 \mathrm{mg} / \mathrm{Kg} \text {, } \\
40 \mathrm{mg} / \mathrm{Kg}\end{array}$ & IP & $28 \mathrm{~d}$ & $\begin{array}{l}\text { proges- } \\
\text { terone }\end{array}$ & $\begin{array}{l}\text { Se- } \\
\text { rum }\end{array}$ & NA & $\begin{array}{l}\text { Radio } \\
\text { imune } \\
\text { essay }\end{array}$ & $\begin{array}{c}\text { Tproges- } \\
\text { terone2X } \\
\text { low dose, } \\
3 \mathrm{X} \text { high } \\
\text { dose SPD }\end{array}$ \\
\hline
\end{tabular}




\begin{tabular}{|c|c|c|c|c|c|c|c|c|c|c|c|c|c|}
\hline $\begin{array}{l}\text { Taketa } \\
\text { et al. } \\
2011\end{array}$ & $\begin{array}{l}\text { Ja- } \\
\text { pan }\end{array}$ & Yes & $\begin{array}{l}\text { Longitu- } \\
\text { dinal } \\
\text { Animal, } \\
\text { con- } \\
\text { trolled }\end{array}$ & $\begin{array}{l}\text { Sprague- } \\
\text { Dawley } \\
\text { rats(F) } \\
\text { rats }\end{array}$ & $\begin{array}{c}\mathrm{n}=4-7 \text { per } \\
\text { group }\end{array}$ & $\begin{array}{l}\text { Sulpiride } \\
100 \mathrm{mg} / \mathrm{Kg}\end{array}$ & $\begin{array}{l}\text { Ga- } \\
\text { vage }\end{array}$ & $\begin{array}{c}2 \\
\text { weeks }\end{array}$ & $\begin{array}{l}\text { Proges- } \\
\text { terone }\end{array}$ & $\begin{array}{l}\text { Se- } \\
\text { rum }\end{array}$ & NA & $\begin{array}{c}\text { Radio } \\
\text { imune } \\
\text { essay }\end{array}$ & $\begin{array}{c}\uparrow 2.1 \mathrm{X} \\
\text { progester } \\
\text { one }\end{array}$ \\
\hline $\begin{array}{l}\text { Dano- } \\
\text { vich } \\
\text { et al., } \\
2008\end{array}$ & $\begin{array}{l}\text { Isra- } \\
\text { el }\end{array}$ & Yes & $\begin{array}{l}\text { Longitu- } \\
\text { dinal } \\
\text { Animal, } \\
\text { con- } \\
\text { trolled }\end{array}$ & $\begin{array}{c}\text { Sprague- } \\
\text { Dawley(M) } \\
\text { rats+ } \\
\text { cell } \\
\text { culture }\end{array}$ & $\begin{array}{l}\mathrm{N}=5 \text { per } \\
\text { group }\end{array}$ & $\begin{array}{c}\text { Clozap- } \\
\text { ine(20mg/ } \\
\text { Kg), tiori- } \\
\text { dazine(20mg/ } \\
\text { Kg), sulpir- } \\
\text { ide(20mg/ } \\
\text { Kg), risperi- } \\
\text { done(0,5mg/ } \\
\text { Kg) }\end{array}$ & IP & $21 \mathrm{~d}$ & $\begin{array}{l}\text { Testos- } \\
\text { terone }\end{array}$ & $\begin{array}{l}\text { Se- } \\
\text { rum }\end{array}$ & NA & $\begin{array}{l}\text { Solid } \\
\text { phase } \\
\text { Radio } \\
\text { imune } \\
\text { essay }\end{array}$ & $\begin{array}{c}\text { No chang- } \\
\text { es }\end{array}$ \\
\hline $\begin{array}{l}\text { Marx } \\
\text { et al., } \\
2006 \mathrm{~b}\end{array}$ & USA & Yes & $\begin{array}{l}\text { Longi- } \\
\text { tudinal, } \\
\text { placebo } \\
\text { con- } \\
\text { trolled }\end{array}$ & $\begin{array}{l}\text { Sprague- } \\
\text { Dawley(M) } \\
\text { rats }\end{array}$ & $\begin{array}{c}n=8-11 \text { por } \\
\text { grupo }\end{array}$ & $\begin{array}{l}\text { Olanzap- } \\
\text { ine(5-10mg/ } \\
\text { Kg), fluoxet- } \\
\text { ine(10-20mg/ } \\
\text { Kg), combina- } \\
\text { tion }\end{array}$ & IP & $\begin{array}{l}5 \mathrm{~d} \\
\text { (euth- } \\
\text { anized } \\
\text { after } \\
1 \mathrm{~h} \text { of } \\
\text { the } \\
\text { dose) }\end{array}$ & $\begin{array}{c}\text { Preg- } \\
\text { neno- } \\
\text { lone, } \\
\text { allo- } \\
\text { preg- } \\
\text { neno- } \\
\text { lone }\end{array}$ & $\begin{array}{l}\text { Se- } \\
\text { rum, } \\
\text { brain }\end{array}$ & $\begin{array}{l}\text { Hip- } \\
\text { po- } \\
\text { cam- } \\
\text { pus }\end{array}$ & $\begin{array}{l}\text { Gas } \\
\text { chro- } \\
\text { ma- } \\
\text { thog- } \\
\text { raphy/ } \\
\text { Mass } \\
\text { espec- } \\
\text { trosco- } \\
\text { py }\end{array}$ & $\begin{array}{c}\text { } \text { allopreg- } \\
\text { nenolone } \\
\text { e hippo- } \\
\text { campus } \\
\text { pregneno- } \\
\text { lone }\end{array}$ \\
\hline $\begin{array}{l}\text { Marx et } \\
\text { al., } 2006\end{array}$ & USA & Yes & $\begin{array}{l}\text { Longi- } \\
\text { tudinal, } \\
\text { placebo } \\
\text { con- } \\
\text { trolled }\end{array}$ & $\begin{array}{l}\text { Sprague- } \\
\text { Dawley(M) } \\
\text { rats }\end{array}$ & $\begin{array}{c}n=6-10 \text { per } \\
\text { group }\end{array}$ & $\begin{array}{c}\text { Clozap- } \\
\text { ine(20mg/ } \\
\text { Kg), Olanzap- } \\
\text { ine(5mg/Kg), } \\
\text { quetiapine } \\
20 \mathrm{mg} / \mathrm{Kg}, \\
\text { aripiprazol } \\
(5 \mathrm{mg} / \mathrm{Kg})\end{array}$ & IP & $\begin{array}{l}1 \mathrm{~d} \\
\text { (euth- } \\
\text { anized } \\
\text { after } \\
1 \mathrm{~h} \text { of } \\
\text { the } \\
\text { dose) }\end{array}$ & $\begin{array}{l}\text { Preg- } \\
\text { neno- } \\
\text { lone }\end{array}$ & $\begin{array}{l}\text { Se- } \\
\text { rum, } \\
\text { brain }\end{array}$ & $\begin{array}{c}\text { serum, } \\
\text { hippo- } \\
\text { cam- } \\
\text { pus, } \\
\text { cortex }\end{array}$ & $\begin{array}{l}\text { Gas } \\
\text { chro- } \\
\text { ma- } \\
\text { thog- } \\
\text { raphy/ } \\
\text { Mass } \\
\text { espec- } \\
\text { trosco- } \\
\text { py }\end{array}$ & $\begin{array}{c}\text { Tpreg- } \\
\text { nenolone } \\
\text { Clozapine } \\
\text { and mild } \uparrow \\
\text { with olan- } \\
\text { zapine }\end{array}$ \\
\hline $\begin{array}{l}\text { Ritsner } \\
\text { et al., } \\
2006\end{array}$ & $\begin{array}{c}\text { Isra- } \\
\text { el }\end{array}$ & Yes & $\begin{array}{l}\text { Longi- } \\
\text { tudinal, } \\
\text { case-con- } \\
\text { trol }\end{array}$ & $\begin{array}{c}\mathrm{Hu}- \\
\text { mans(M) } \\
\text { Coleta } \\
8-9 \mathrm{~h}\end{array}$ & $\begin{array}{c}\mathrm{n}=21,14 \\
\text { controls }\end{array}$ & Non specified & Oral & $\begin{array}{c}8 \\
\text { weeks }\end{array}$ & $\begin{array}{l}\text { DHEA, } \\
\text { DHEA-S, } \\
\text { andro- } \\
\text { stene- } \\
\text { dione e } \\
\text { testos- } \\
\text { terone }\end{array}$ & $\begin{array}{l}\text { Se- } \\
\text { rum }\end{array}$ & NA & $\begin{array}{l}\text { Radio } \\
\text { imune } \\
\text { essay }\end{array}$ & $\begin{array}{c}\text { No chang- } \\
\text { es }\end{array}$ \\
\hline $\begin{array}{l}\text { Ritsner } \\
\text { et al., } \\
2004\end{array}$ & $\begin{array}{l}\text { Isra- } \\
\text { el }\end{array}$ & Sim & $\begin{array}{l}\text { Longitu- } \\
\text { dinal, } \\
\text { case- } \\
\text { control }\end{array}$ & $\begin{array}{c}\text { Humans } \\
(\mathrm{M}=38) \\
\text { Coleta } \\
8-9 \mathrm{~h}\end{array}$ & $\begin{array}{c}\mathrm{n}=40,15 \\
\text { controls }\end{array}$ & $\begin{array}{l}\text { Non } \\
\text { specified. With } \\
\text { exclusion of } \\
\text { the use of } \\
\text { other psycho- } \\
\text { tropics. }\end{array}$ & Oral & $\begin{array}{c}2 \\
\text { weeks }\end{array}$ & $\begin{array}{l}\text { DHEA, } \\
\text { DHEA-S }\end{array}$ & $\begin{array}{l}\text { Se- } \\
\text { rum }\end{array}$ & NA & $\begin{array}{l}\text { Radio } \\
\text { imune } \\
\text { essay }\end{array}$ & $\begin{array}{c}\text { No chang- } \\
\text { es }\end{array}$ \\
\hline $\begin{array}{l}\text { Monten- } \\
\text { leone et } \\
\text { al., } 2004\end{array}$ & Italy & Yes & $\begin{array}{l}\text { Longi- } \\
\text { tudinal, } \\
\text { cross } \\
\text { control }\end{array}$ & $\begin{array}{l}\text { Humans } \\
(\mathrm{M}=5, \mathrm{~F}=4) \\
\text { Coleta } \\
8-9 \mathrm{~h}\end{array}$ & $\mathrm{~N}=9$ & $\begin{array}{l}\text { Clozapine } \\
\text { (600mg/dia) }\end{array}$ & Oral & $\begin{array}{c}24 \\
\text { weeks }\end{array}$ & $\begin{array}{l}\text { Allo- } \\
\text { preg- } \\
\text { nano- } \\
\text { lone, } \\
\text { THDOC }\end{array}$ & $\begin{array}{l}\text { Se- } \\
\text { rum }\end{array}$ & NA & $\begin{array}{c}\text { Radio } \\
\text { imune } \\
\text { essay }\end{array}$ & $\begin{array}{c}\text { No chang- } \\
\text { es }\end{array}$ \\
\hline $\begin{array}{l}\text { Huber } \\
\text { et al., } \\
2004\end{array}$ & $\begin{array}{l}\text { Ger- } \\
\text { many }\end{array}$ & Yes & $\begin{array}{l}\text { Longi- } \\
\text { tudinal, } \\
\text { case-con- } \\
\text { trol }\end{array}$ & $\begin{array}{c}\mathrm{Hu}- \\
\text { mans(F) } \\
\text { Coleta } \\
\text { 9-10h30 }\end{array}$ & $n=50$ & Non specified & Oral & $\begin{array}{c}4 \\
\text { weeks }\end{array}$ & $\begin{array}{l}\text { Proges- } \\
\text { terone }\end{array}$ & $\begin{array}{l}\text { Se- } \\
\text { rum }\end{array}$ & NA & $\begin{array}{l}\text { Radio } \\
\text { imune } \\
\text { essay }\end{array}$ & $\begin{array}{c}\text { No chang- } \\
\text { es }\end{array}$ \\
\hline $\begin{array}{l}\text { Taheri- } \\
\text { anfard } \\
\text { et al., } \\
2004\end{array}$ & Iran & Yes & $\begin{array}{l}\text { Longi- } \\
\text { tudinal, } \\
\text { case-con- } \\
\text { trol }\end{array}$ & $\begin{array}{l}\text { Humans } \\
\text { (M) Coleta } \\
8 \mathrm{~h}\end{array}$ & $\mathrm{~N}=49$ & Non specified & Oral & $\begin{array}{c}6 \\
\text { weeks }\end{array}$ & $\begin{array}{l}\text { Proges- } \\
\text { terone, } \\
\text { testos- } \\
\text { terone }\end{array}$ & $\begin{array}{l}\text { Se- } \\
\text { rum }\end{array}$ & NA & ELISA & $\begin{array}{c}\text { Tproges- } \\
\text { terone }\end{array}$ \\
\hline $\begin{array}{c}\text { Nech- } \\
\text { mad et } \\
\text { al., } 2003\end{array}$ & $\begin{array}{l}\text { Isra- } \\
\text { el }\end{array}$ & Yes & $\begin{array}{l}\text { Longi- } \\
\text { tudinal, } \\
\text { placebo } \\
\text { con- } \\
\text { trolled }\end{array}$ & $\begin{array}{l}\text { Sprague- } \\
\text { Dawley(M) } \\
\text { rats }\end{array}$ & $\begin{array}{c}\mathrm{N}=8-9 \text { per } \\
\text { group }\end{array}$ & $\begin{array}{c}\text { Clozap- } \\
\text { ine(5-15mg/ } \\
\mathrm{Kg}), \text { Haloperi- } \\
\text { dol(1MG/Kg) }\end{array}$ & IP & $\begin{array}{l}8 \mathrm{~d} \\
\text { (euth- } \\
\text { aniz- } \\
\text { ed } \\
\text { after } \\
2 \mathrm{~h} \text { of } \\
\text { the } \\
\text { dose) }\end{array}$ & $\begin{array}{l}\text { DHEA, } \\
\text { DHEA-S }\end{array}$ & Brain & Cortex & $\begin{array}{l}\text { Radio } \\
\text { immune } \\
\text { essay }\end{array}$ & $\begin{array}{c}\downarrow D H E A \text { e } \\
\text { DHEA-S } \\
\text { clozapine, } \\
\text { no chang- } \\
\text { es with } \\
\text { haloper- } \\
\text { idol }\end{array}$ \\
\hline
\end{tabular}




\begin{tabular}{|c|c|c|c|c|c|c|c|c|c|c|c|c|c|}
\hline $\begin{array}{l}\text { Freye } \\
\text { and } \\
\text { Seliga, } \\
2003\end{array}$ & USA & Yes & $\begin{array}{l}\text { Longi- } \\
\text { tudinal, } \\
\text { placebo } \\
\text { con- } \\
\text { trolled }\end{array}$ & $\begin{array}{l}\text { Long-Ev- } \\
\text { ans (F) } \\
\text { rats }\end{array}$ & $\mathrm{N}=55$ & 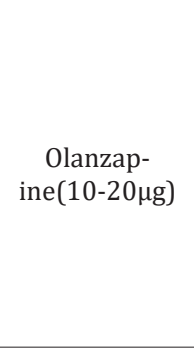 & $\begin{array}{l}\text { Intra- } \\
\text { cere- } \\
\text { bral } \\
\text { (VTA) }\end{array}$ & $\begin{array}{c}3 \\
\text { weeks }\end{array}$ & $\begin{array}{l}\text { 3a,5a- } \\
\text { THP, } \\
\text { Proges- } \\
\text { terone }\end{array}$ & $\begin{array}{l}\text { Se- } \\
\text { rum, } \\
\text { brain }\end{array}$ & $\begin{array}{l}\text { Mes- } \\
\text { en- } \\
\text { cepha- } \\
\text { lon }\end{array}$ & $\begin{array}{l}\text { Radio } \\
\text { immune } \\
\text { essay }\end{array}$ & $\begin{array}{c}\text { No } \\
\text { changes in } \\
\text { sérum.No } \\
\text { changes in } \\
\text { proges- } \\
\text { terona } \\
\text { in VTA. } \\
\uparrow 3 a, 5 a- \\
\text { THP } \\
\text { (VTA) }\end{array}$ \\
\hline $\begin{array}{l}\text { Freye } \\
\text { and } \\
\text { Seliga, } \\
2003 b\end{array}$ & USA & Yes & $\begin{array}{l}\text { Longi- } \\
\text { tudinal, } \\
\text { placebo } \\
\text { con- } \\
\text { trolled } \\
\end{array}$ & $\begin{array}{l}\text { Long-Ev- } \\
\text { ans (F) } \\
\text { rats }\end{array}$ & $\begin{array}{c}\mathrm{N}=33 \mathrm{n}=9( \\
\text { evalu } \\
\text {-ated leves) }\end{array}$ & $\begin{array}{l}\text { Olanzap- } \\
\text { ine(5-10mg/ } \\
\mathrm{Kg})\end{array}$ & IP & $1 \mathrm{~d}$ & $\begin{array}{l}\text { 3a,5a- } \\
\text { THP, } \\
\text { Proges- } \\
\text { terone }\end{array}$ & $\begin{array}{l}\text { Se- } \\
\text { rum, } \\
\text { cére- } \\
\text { brain }\end{array}$ & $\begin{array}{l}\text { Whole } \\
\text { brain }\end{array}$ & $\begin{array}{l}\text { Radio } \\
\text { immune } \\
\text { essay }\end{array}$ & $\begin{array}{c}\uparrow 3 a, 5 a- \\
\text { THP, } \\
\text { Progester- } \\
\text { one, brain } \\
\text {, serum }\end{array}$ \\
\hline $\begin{array}{l}\text { Marx et } \\
\text { al., } 2003\end{array}$ & USA & Yes & $\begin{array}{l}\text { Longi- } \\
\text { tudinal, } \\
\text { placebo } \\
\text { con- } \\
\text { trolled }\end{array}$ & $\begin{array}{l}\text { Sprague- } \\
\text { Dawley(M) } \\
\text { rats }\end{array}$ & $\begin{array}{c}n=6-11 \text { per } \\
\text { group }\end{array}$ & $\begin{array}{c}\text { olanzapine } \\
(2.5-10.0 \mathrm{mg} / \\
\mathrm{kg}), \text { clozapine } \\
(5.0-20.0 \mathrm{mg} / \\
\mathrm{kg}), \text { risperi- } \\
\text { done }(0.1-1.0 \\
\mathrm{mg} / \mathrm{kg}) \\
\text { haloperidol } \\
(0.1-1.0 \mathrm{mg} / \\
\mathrm{kg})\end{array}$ & IP & $1 \mathrm{~d}$ & $\begin{array}{c}\text { Allo- } \\
\text { preg- } \\
\text { nano- } \\
\text { lone } \\
\text { (brain), } \\
\text { proges- } \\
\text { terone } \\
\text { (serum) }\end{array}$ & $\begin{array}{l}\text { Se- } \\
\text { rum, } \\
\text { brain }\end{array}$ & cortex & $\begin{array}{l}\text { Radio } \\
\text { immune } \\
\text { essay }\end{array}$ & $\begin{array}{c}\text { } \text { allopreg- } \\
\text { nanolone, } \\
\text { sérum } \\
\text { and brain } \\
\text { progester- } \\
\text { one with } \\
\text { olanzap- } \\
\text { ine and } \\
\text { clozapine. } \\
\text { No chang- } \\
\text { es halo- } \\
\text { peridol/ } \\
\text { risperi- } \\
\text { done }\end{array}$ \\
\hline $\begin{array}{l}\text { Baptista } \\
\text { et al., } \\
2002\end{array}$ & $\begin{array}{l}\text { Can- } \\
\text { ada }\end{array}$ & Yes & $\begin{array}{l}\text { Longi- } \\
\text { tudinal, } \\
\text { placebo } \\
\text { con- } \\
\text { trolled }\end{array}$ & Wistar rats & $\begin{array}{c}\mathrm{N}=648 \text { per } \\
\text { group }\end{array}$ & $\begin{array}{c}\text { Risperi- } \\
\text { done(0,5mg/ } \\
\text { Kg), sulpiri- } \\
\operatorname{de}(20 \mathrm{mg} / \mathrm{Kg})\end{array}$ & SC & $12 \mathrm{~d}$ & $\begin{array}{l}\text { Proges- } \\
\text { terone }\end{array}$ & $\begin{array}{l}\text { Se- } \\
\text { rum }\end{array}$ & NA & $\begin{array}{l}\text { Radio } \\
\text { immune } \\
\text { essay }\end{array}$ & $\begin{array}{l}\text { No chang- } \\
\text { es }\end{array}$ \\
\hline $\begin{array}{c}\text { Dona- } \\
\text { deu \& } \\
\text { Thomp- } \\
\text { son, } \\
2002\end{array}$ & USA & & $\begin{array}{l}\text { Longi- } \\
\text { tudinal, } \\
\text { placebo } \\
\text { con- } \\
\text { trolled }\end{array}$ & Mares & $\mathrm{N}=14$ & $\begin{array}{l}\text { Sulpiride } \\
1 \mathrm{mg} / \mathrm{Kg}\end{array}$ & SC & $30 \mathrm{~d}$ & $\begin{array}{l}\text { Proges- } \\
\text { terone }\end{array}$ & $\begin{array}{l}\text { Se- } \\
\text { rum }\end{array}$ & NA & $\begin{array}{c}\text { Radio } \\
\text { imune } \\
\text { essay }\end{array}$ & $\begin{array}{c}\text { No chang- } \\
\text { es }\end{array}$ \\
\hline $\begin{array}{l}\text { Barbac- } \\
\text { cia et al., } \\
2001\end{array}$ & Italy & Yes & $\begin{array}{l}\text { Longi- } \\
\text { tudinal, } \\
\text { placebo } \\
\text { con- } \\
\text { trolled }\end{array}$ & $\begin{array}{l}\text { Sprague- } \\
\text { Dawley(M) } \\
\text { rats }\end{array}$ & $\begin{array}{c}n=8-10 \text { per } \\
\text { group }\end{array}$ & $\begin{array}{c}\text { Clozapine } \\
1.25,2.5,5,10 \text {, } \\
\text { or } 20 \mathrm{mg} / \mathrm{Kg} \text {, } \\
\text { haloperidol } 0.1 \\
\text { or } 0.5 \mathrm{mg} / \mathrm{kg}\end{array}$ & IP & $\begin{array}{l}1 \mathrm{~d}(45 \\
\min , \\
3 \mathrm{~h}) \\
19 \mathrm{~d}\end{array}$ & $\begin{array}{c}\text { Proges- } \\
\text { terone, } \\
\text { THDOC, } \\
\text { allo- } \\
\text { preg- } \\
\text { nano- } \\
\text { lone }\end{array}$ & $\begin{array}{l}\text { Se- } \\
\text { rum, } \\
\text { brain }\end{array}$ & $\begin{array}{l}\text { Cor- } \\
\text { tex, } \\
\text { stria- } \\
\text { tum }\end{array}$ & $\begin{array}{l}\text { Gas } \\
\text { chro- } \\
\text { ma- } \\
\text { thog- } \\
\text { raphy/ } \\
\text { Mass } \\
\text { espec- } \\
\text { trosco- } \\
\text { py }\end{array}$ & $\begin{array}{c}\uparrow A P, \\
\text { THDOC< } \\
\text { progester- } \\
\text { one com } \\
\text { clozapine } \\
\text { (10mg/ } \\
\text { Kg) serum } \\
\text { and brain } \\
\text { Normal } \\
\text { after 3h. } \\
\text { No chang- } \\
\text { es with } \\
\text { haloper- } \\
\text { idol }\end{array}$ \\
\hline $\begin{array}{l}\text { Marx et } \\
\text { al., } 2000\end{array}$ & USA & Yes & $\begin{array}{l}\text { Longi- } \\
\text { tudinal, } \\
\text { placebo } \\
\text { con- } \\
\text { trolled }\end{array}$ & $\begin{array}{l}\text { Sprague- } \\
\text { Dawley(M) } \\
\text { rats }\end{array}$ & $\begin{array}{l}\mathrm{N}=10-11 \\
\text { per group }\end{array}$ & $\begin{array}{c}\text { Olanzapine } \\
(0,2.5,5.0, \text { or } \\
10.0 \mathrm{mg} / \mathrm{kg})\end{array}$ & IP & $1 \mathrm{~d}$ & $\begin{array}{l}\text { Allo- } \\
\text { preg- } \\
\text { nano- } \\
\text { lone- } \\
\text { cortical, } \\
\text { proges- } \\
\text { terone } \\
\text {-serum }\end{array}$ & $\begin{array}{l}\text { Se- } \\
\text { rum, } \\
\text { brain }\end{array}$ & Cortex & $\begin{array}{l}\text { Radio } \\
\text { imune } \\
\text { essay }\end{array}$ & $\begin{array}{l}\text { Talopreg- } \\
\text { nanolone } \\
\text { e proges- } \\
\text { terone }\end{array}$ \\
\hline $\begin{array}{l}\text { Baptis- } \\
\text { ta, } 1999\end{array}$ & $\begin{array}{l}\text { Ven- } \\
\text { ezu- } \\
\text { ela }\end{array}$ & Yes & $\begin{array}{l}\text { Longi- } \\
\text { tudinal, } \\
\text { placebo } \\
\text { con- } \\
\text { trolled }\end{array}$ & Humans & $\begin{array}{c}\mathrm{N}=45 \mathrm{~Pa}- \\
\text { cientss=18, } \\
\text { controls=27 }\end{array}$ & Non specified & Oral & $30 \mathrm{~d}$ & $\begin{array}{c}\text { DHEA-S, } \\
\text { proges- } \\
\text { terone }\end{array}$ & $\begin{array}{l}\text { Se- } \\
\text { rum }\end{array}$ & NA & $\begin{array}{l}\text { Radio } \\
\text { immune } \\
\text { essay }\end{array}$ & $\begin{array}{c}\downarrow \text { proges- } \\
\text { terone (F), } \\
\downarrow \text { Testos- } \\
\text { terone e } \\
\text { DHEA-S } \\
\text { (M) }\end{array}$ \\
\hline $\begin{array}{l}\text { Baptista } \\
\text { et al., } \\
2001\end{array}$ & $\begin{array}{l}\text { Ven- } \\
\text { ezu- } \\
\text { ela }\end{array}$ & Yes & $\begin{array}{l}\text { transver- } \\
\text { sal, con- } \\
\text { trolled }\end{array}$ & Humans & $\begin{array}{l}\mathrm{N}=26,26 \\
\text { controls }\end{array}$ & Non specified & Oral & NA & $\begin{array}{l}\text { Proges- } \\
\text { terone }\end{array}$ & $\begin{array}{l}\text { Se- } \\
\text { rum }\end{array}$ & NA & $\begin{array}{l}\text { Radio } \\
\text { immune } \\
\text { essay }\end{array}$ & $\begin{array}{l}\downarrow \text { Proges- } \\
\text { terone } \\
\text { after AP }\end{array}$ \\
\hline
\end{tabular}




\begin{tabular}{|c|c|c|c|c|c|c|c|c|c|c|c|}
\hline $\begin{array}{c}\text { Baptista } \\
\text { et al., } \\
1997\end{array}$ & $\begin{array}{c}\text { Ven- } \\
\text { ezu- } \\
\text { ela }\end{array}$ & Yes & $\begin{array}{c}\text { Longi- } \\
\text { tudinal, } \\
\text { placebo } \\
\text { con- } \\
\text { trolled }\end{array}$ & Humans & N=28 & $\begin{array}{c}\text { Sulpiride } \\
(200 \mathrm{mg} / \text { day })\end{array}$ & Oral & $30 \mathrm{~d}$ & $\begin{array}{c}\text { Proges- } \\
\text { terone }\end{array}$ & $\begin{array}{c}\text { Se- } \\
\text { rum }\end{array}$ & $\begin{array}{c}\text { Nadio } \\
\text { immune } \\
\text { essay }\end{array}$ \\
$\begin{array}{c}\text { No chang- } \\
\text { es }\end{array}$ \\
\hline
\end{tabular}

It was identified a total of 291 studies through our search strategy. Of these studies, 20 were included in our review. A further search throughout the reference list yielded one more study. In the end, a total of 21 studies were included in our review, with further details available in Table 1 . Of these 21 studies, 13 were animal studies, with the rest evaluating neuroactive steroids levels in humans. Three of these studies performed HPLC associated with another essay to measure amino acids (e.g. spectroscopy), and the other 14 studies used only HPLC analysis to measure the amino acid levels. Five studies used post mortem material for the analysis, with the other 12 studies analyzing microdyalisated samples of in vivo specimens. Only one study didn't presented a placebo control group, utilizing a basal measure to control their data. Fourteen studies used Sprague-Dale rats, one study utilized Hooded Lister rats, another study used wistar rats and only one study didn't stated which type of rat was used. The number of rats per group varied from 6 to 17 for each group. The time from drug initiation and tissue analysis varied from 60 minutes to 8 months (Table 1).

\section{Antipsychotic agents}

A total of seven different antipsychotic agents were evaluated in the studies included in the present review (haloperidol, sulpiride, clozapine, olanzapine, risperidone, quetiapine and aripiprazol.), although some studies enrolled patients in use of antipsychotics that weren't specified. All the human studies used the medication orally. Among the animal studies, nine administered the antipsychotic through intraperitoneal (i.p.) injections, two through subcutaneous (s.c.) injections, one through oral administration (gavage) and one used intrathecal injections (i.t.).

\section{Neuroactive steroids}

Throughout the studies selected to take part of this review, a wide range of neuroactive steroids were studied. We found studies that evaluated the following neuroactive steroids: progesterone, allopregnanolone, DHEA, DHEA-S, TDHOC, pregnenolone and 3a,5a-THP.

\section{Human studies}

Changes in basal neuroactive steroids levels after antipsychotic use. It was observed a wide variation of changes among the different studies present in this review, above all among the human studies. At least part of this variation was related to methodological issues, for instance, the lack of control over the type of antipsychotic used by the patients enrolled in the studies. Only two of the total (eight) of human studies reviewed here controlled the type of antipsychotic used, one using clozapine and the other study sulpiride (MONTENLEONE et al., 2004; BAPTISTA et al., 1997). Therefore, it is hard to make clearly conclusions with the data from these human reviewed here, with these data had demonstrated diverse and non-conclusive alterations on the plasma levels of progesterone, no changes on allopregnanolone and TDHOC levels after clozapine use. Three human studies evaluated DHEA/DHEA-S levels, with no alterations in two of these, and decreased levels in the third study, although there was no control regarding the type of antipsychotic used by the patients enrolled in these studies.

\section{Animal studies}

Changes in basal neuroactive steroids levels after antipsychotic use. All animal studies controlled the type of antipsychotic used, different form the majority of human studies. However, the difference among the studies regarding how the medication was administered apparently influenced the observed results. Among the animal studies, nine administered the antipsychotic through intraperitoneal injections (IP). Two used subcutaneous injections (SC), one used medication given orally by gavage and the last study utilized intracerebral injections. Of 13 animal studies that evaluated the levels of neuroactive steroids, nine of these measured progesterone levels, with no changes in three studies, and increased plasma levels in six studies. Among the three studies with negative results, the antipsychotic used were sulpiride in lower doses $11 \mathrm{mg}$ / kg, 20mg / kg), risperidone, haloperidol and olanzapine, this last one administered through intracerebral injections (FREYE AND SELIGA, 2003). The average duration of antipsychotic use was 15,75 days. Among the six studies that showed increased levels of progesterone, the antipsychotic used were: sulpiride in high dose (40mg / kg), clozapine and olanzapine (IP gavage, SC). The average of antipsychotic use was 10.6 days. The same researchers investigated in two studies the pregnenolone brain levels in rats, showing that clozapine and olanzapine acutely increased the levels of pregnenolone in rats $[10,11]$. Four studies evaluating allopregnanolone showed increased brain levels after the use of olanzapine and clozapine. No changes with haloperidol or risperidone were observed $[3,9,12,13]$. The only animal study that investigated DHEA/DHEA-S levels, showed that clozapine decreased brain levels of DHEA-S, unlike haloperidol [14]. Increased TDHOC levels after use of clozapine were observed in the only study that measured this neuroactive steroid [5]. Two studies from the same group showed increased brain levels of 3a,5a-THP after olanzapine use $[15,16]$.

\section{Discussion}

There is emerging preclinical and clinical evidence supporting a role for neurosteroids in the neurobiology and therapeutics of schizophrenia, with a primary focus on progesterone, pregnenolone and selected pregnenolone metabolites-specifically pregnenolone sulfate, a neurosteroid that positively modulates excitatory glutamatergic NMDA receptors and allopregnanolone, a positive modulator of inhibitory GABAA receptors. The positive modulation of NMDA receptors by pregnenolone sulfate could 
potentially contribute to the amelioration of hypothesized NMDA receptor hypofunction in schizophrenia (JAVITT, 2004; MILLAN, 2005; RUJESCU et al., 2006; JAVITT, 2007) [17]. Metabolism to allopregnanolone could impact the GABA neurotransmitter system, which also appears to be dysregulated in schizophrenia (BENES AND BERRETTA, 2001; LEWIS et al., 2003, 2004, 2005; GUIDOTTI et al., 2005; BENES et al., 2007), as allopregnanolone potentiates GABAA receptor responses more potently than benzodiazepines or barbiturates (MAJEWSKA et al., 1986; MORROW et al., 1987, 1990). Different patterns were observed throughout the studies in our review, concerning the alterations of neuroactive steroids brain and serum levels after the use of antipsychotic agents. It is of great value to carefully analyze this issue since the increasing attention over neuroactive steroids in schizophrenia pathophysiology and treatment has gained increasing attention in the past decade (MARX et al., 2011). Multiple lines of evidence suggest that alterations in peripheral neuroactive steroids may be linked to brain functions and that changes in steroids metabolism could play a role in the pathogenesis of psychiatric disorders, like schizophrenia [18]. Even with all this attention over these alterations involving neuroactive steroids, we didn't find enough data among the studies included in our review that support consistent changes with the use of antipsychotics in neuroactive steroids content in human serum samples. Among the 08 studies here reviewed, only two have controlled the type of antipsychotic used, one using clozapine and the other sulpiride (MONTENLEONE et al., 2004) [19]. Therefore, it is hard to make clearly conclusions with the data from these human studies, with these data had demonstrated diverse and non-conclusive alterations on the plasma levels of progesterone, no changes on allopregnanolone and TDHOC levels after clozapine use. Another point of interest among researchers is the hypothesis that neuroactive steroids changes after antipsychotic use could explain the differences among the different classes of these agents, believing that not only dopamine and serotonin changes explain the atypical profile of some antipsychotics, and their lower propensity to cause motor side effects. Also noteworthy is the particularly important role of progesterone in psychosis, with recent data pointing to progesterone levels being inversely associated with the severity of positive symptoms in first episode psychosis [20-26]. It was of great value the observation that the progesterone levels measured in the animal studies here reviewed had showed a clear differentiation between first and second generation antipsychotic medications, with the latter showing the capability of change the progesterone levels, what wasn't possible with the use of haloperidol, for example [9, 27-39].

\section{Conclusion}

The description of the findings of the studies included in this review, suggest that there are sufficient studies to help demonstrate a relationship between antipsychotic use and changes in brain and serum levels of neuroactive steroids, mainly when we consider animal studies. Nevertheless, the heterogeneity of findings and lack of consistency in the observed changes difficult the formulation of definitive conclusions about this issue, pointing to the need of more studies that help to elucidate the remaining answered questions about the role of antipsychotic medications in changing neuroactive steroids content in humans. The antipsychotic effects observed on neuroactive steroids levels reinforce their potential role in the pathophysiology of mental disorders, particularly schizophrenia. In the case of progesterone, the studies are in sufficient number to demonstrate the effect of antipsychotic on changes in their levels in animal and human studies. Both clozapine and olanzapine showed to be capable of altering the levels of progesterone. For the other neuroactive steroids, the inconclusive data points to the need for more well designed studies, with better controlled variables, regarding type of antipsychotic used for example, or studies using the medication for longer period of time (more than 30 days).

\section{Acknowledgement}

None.

\section{Conflict of Interest}

\section{No conflict of interest.}

\section{References}

1. Helio Elkis, Herbert Y Meltzer (2007) Esquizofrenia refratária. Rev Bras Psiquiatr 29(2): 41-47.

2. Szkultecka Dębek M, Miernik K, Stelmachowski J, Jakovljević M, Jukić V, et al. (2016) Schizophrenia causes significant burden to patients' and caregivers' lives. Psychiatr Danub 28(2): 104-110.

3. Tandon R, Carpenter WT, Davis JM (2007) First- and second-generation antipsychotics: learning from CUtLASS and CATIE. Arch Gen Psychiatry 64(8): 977-978.

4. Howes OD, Kapur S (2009) The dopamine hypoyhesis of schizophrenia: version III-the final common pathway. Schizophr Bull 35(3): 549-562.

5. Faden J, Citrome L (2019) Resistance is not futile: treatment-refractory schizophrenia - overview, evaluation and treatment. Expert Opin Pharmacother 20(1): 11-24.

6. Yang AC, Tsai SJ (2017) New Targets for Schizophrenia Treatment beyond the Dopamine Hypothesis. Int J Mol Sci 18(8).

7. Misiak B, Frydecka D, Loska O, Moustafa AA, Samochowiec J, et al. (2018) Testosterone, DHEA and DHEA-S in patients with schizophrenia: A systematic review and meta-analysis. Psychoneuroendocrinology 89: 92-102.

8. Nunes EA, MacKenzie EM, Rossolatos D, Perez-Parada J, Baker GB, et al. (2012) D-serine and schizophrenia: an update. Expert Rev Neurother 12(7): 801-812.

9. Marx CE, VanDoren MJ, Duncan GE, Lieberman JA, Morrow AL (2003) Olanzapine and clozapine increase the GABAergic neuroactive steroid allopregnanolone in rodents. Neuropsychopharmacology 28(1): 1-13.

10. Marx CE, Shampine LJ, Duncan GE, VanDoren MJ, Grobin AC, et al. (2006) Clozapine markedly elevates pregnenolone in rat hippocampus, cerebral cortex, and serum: candidate mechanism for superior efficacy? Pharmacol Biochem Behav 84(4): 598-608.

11. Marx CE, Shampine LJ, Khisti RT, Trost WT, Bradford DW, et al. (2006) Olanzapine and fluoxetine administration and coadministration increase rat hippocampal pregnenolone, allopregnanolone and peripheral deoxycorticosterone: implications for therapeutic actions. Pharmacol Biochem Behav 84(4): 609-617.

12. Barbaccia ML, Affricano D, Purdy RH, Maciocco E, Spiga F, et al. (2001) Clozapine, but not haloperidol, increases brain concentrations of neuroactive steroids in the rat. Neuropsychopharmacology 25(4): 489497.

13. Marx CE, Duncan GE, Gilmore JH, Lieberman JA, Morrow AL (2000) Olanzapine increases allopregnanolone in the rat cerebral cortex. Biol Psychiatry 47(11): 1000-1004. 
14. Nechmad A, Maayan R, Ramadan E, Morad O, Poyurovsky M, et al. (2003) Clozapine decreases rat brain dehydroepiandrosterone and dehydroepiandrosterone sulfate levels. Eur Neuropsychopharmacol 13(1): 29-31.

15. Frye C, Seliga A (2003) Effects of olanzapine infusions to the ventral tegmental area on lordosis and midbrain 3alpha,5alpha-THP concentrations in rats. Psychopharmacology (Berl) 170(2): 132-139.

16. Frye CA, Seliga AM (2003) Olanzapine's effects to reduce fear and anxiety and enhance social interactions coincide with increased progestin concentrations of ovariectomized rats. Psychoneuroendocrinology 28(5): 657-673.

17. Coyle JT (2006) Glutamate and schizophrenia: beyond the dopamine hypothesis. Cell. Mol. Neurobiol 26(4-6): 365-384.

18. Cai H, Zhou X, Dougherty GG, Reddy RD, Haas GL, et al. (2018) Pregnenolone-progesterone-allopregnanolone pathway as a potential therapeutic target in first-episode antipsychotic-naïve patients with schizophrenia. Psychoneuroendocrinology 90: 43-51.

19. Baptista T, Molina MG, Martinez JL, de Quijada M, Calanche de Cuesta I, et al. (1997) Effects of the antipsychotic drug sulpiride on reproductive hormones in healthy premenopausal women: relationship with body weight regulation. Pharmacopsychiatry 30(6): 256-262.

20. Belvederi Murri M, Fanelli F, Pagotto U, Bonora E, Triolo F, et al. (2016) Neuroactive Steroids in First-Episode Psychosis: A Role for Progesterone? Schizophr Res Treatment.

21. Baptista T, Araujo de Baptista E, Ying Kin NM, Beaulieu S, Walker D, et al. (2002) Comparative effects of the antipsychotics sulpiride or risperidone in rats. I: bodyweight, food intake, body composition, hormones and glucose tolerance. Brain Res 957(1): 144-151.

22. Baptista T, Lacruz A, Angeles F, Silvera R, de Mendoza S, et al. (2001) Endocrine and metabolic abnormalities involved in obesity associated with typical antipsychotic drug administration. Pharmacopsychiatry 34(6):223-231

23. Baptista T, Reyes D, Hernández L (1999) Antipsychotic drugs and reproductive hormones: relationship to body weight regulation. Pharmacol Biochem Behav 62(3): 409-417.

24. Coyle JT (2003) NMDA receptors and schizophrenia Ann. N.Y. Acad. Sci 1003: 318-327.

25. Danovich L, Veenman L, Leschiner S, Lahav M, Shuster V, et al. (2007) The influence of clozapine treatment and other antipsychotics on the $18 \mathrm{kDa}$ translocator protein, formerly named the peripheraltype benzodiazepine receptor, and steroid production. Eur Neuropsychopharmacol 18(1): 24-33.

26. Donadeu FX, Thompson DL Jr (2002) Administration of sulpiride to anovulatory mares in winter: effects on prolactin and gonadotropin concentrations, ovarian activity, ovulation and hair shedding. Theriogenology 57(2): 963-976.
27. Gaspar PA, Bustamante ML, Silva H, Aboitiz F (2009) Molecular mechanisms underlying glutamatergic dysfunction in schizophrenia: therapeutic implications. J Neurochem 111(4): 891-900.

28. Gordon JA (2010) Testing the glutamate hypothesis of schizophrenia. Nat. Neurosci 13(1): 2-4.

29. Grimm JW, See RE (2000) Chronic haloperidol-induced alterations in pallidal GABA and striatal $\mathrm{D}(1)$-mediated dopamine turnover as measured by dual probe microdialysis in rats. Neuroscience 100(3): 507-514.

30. Grunze HC, Rainnie DG, Hasselmo ME, Barkai E, Hearn EF, et al. (1996) NMDA-dependent modulation of CA1 local circuit inhibition J.Neuroscci 16(6): 2034-2043.

31. Heidbreder CA, Foxton R, Cilia J, Hughes ZA, Shah AJ, et al. (2001) Increased responsiveness of dopamine to atypical, but not typical antipsychotics in the medial prefrontal cortex of rats reared in isolation. Psychopharmacology (Berl) 156(2-3): 338-351.

32. Huber TJ, Borsutzky M, Schneider U, Emrich HM (2004) Psychotic disorders and gonadal function: evidence supporting the oestrogen hypothesis. Acta Psychiatr Scand 109(4): 269-274.

33. Monteleone P, Fabrazzo M, Serra M, Tortorella A, Pisu MG, et al. (2004) Long-term treatment with clozapine does not affect morning circulating levels of allopregnanolone and THDOC in patients with schizophrenia: a preliminary study. J Clin Psychopharmacol 24(4): 437-440.

34. Mostafapour S, Zare S, Sadrkhanlou RA, Ahmadi A, Razi M (2014) Sulpiride-induced hyperprolactinemia in mature female rats: evidence for alterations in the reproductive system, pituitary and ovarian hormones. Int J Fertil Steril 8(2):193-206

35. Ritsner M, Gibel A, Ram E, Maayan R, Weizman A (2006) Alterations in DHEA metabolism in schizophrenia: two-month case-control study. Eur Neuropsychopharmacol 16(2): 137-146.

36. Ritsner M, Maayan R, Gibel A, Strous RD, Modai I, et al. (2004) Elevation of the cortisol/dehydroepiandrosterone ratio in schizophrenia patients. Eur Neuropsychopharmacol 14(4): 267-273.

37. Taherianfard M, Shariaty M (2004) Evaluation of serum steroid hormones in schizophrenic patients. Indian J Med Sci 58(1): 3-9.

38. Taketa Y, Yoshida M, Inoue K, Takahashi M, Sakamoto Y, et al. (2011) Differential stimulation pathways of progesterone secretion from newly formed corpora lutea in rats treated with ethylene glycol monomethyl ether, sulpiride, or atrazine. Toxicol Sci 121(2): 267-278.

39. Yamamura S, Ohoyama K, Hamaguchi T, Kashimoto K, Nakagawa M, et al. (2009) Effects of quetiapine on monoamine, GABA, and glutamate release in rat prefrontal cortex. Psychopharmacology (Berl) 206(2): 243-258. 